Apidologie, 1984, 15 (3), 285-302

\title{
ETUDE BIOMÉTRIQUE DE LA VARIABILITÉ GÉOGRAPHIQUE DES POPULATIONS D'APIS MELLIFERA EN ITALIE SEPTENTRIONALE
}

\author{
Mirella LEPORATI *, Marina VALLI * et Sandro CAVICCHI ** \\ *Istituto Nazionale di Apicoltura, Bologna (Italie) \\ *: Centro di Studio sull'Ecologia e Genetica Quantitativa, \\ Università di Bologna (Italie)
}

\begin{abstract}
RESUME
Une étude biométrique effectuée sur des échantillons d’abeilles prélevées en Ligurie occidentale (frontière avec la France), dans la province de Trento (frontière avec l'Autriche) et dans la région de l'Emilie-Romagne (Italie septentrionale) a mis en évidence que dans cette dernière région seulement la population d'abeilles est homogène et qu'elle appartient à la race Apis mellifera ligustica.

Les abeilles de la Ligurie ressemblent nettement à celles de la race Apis mellifera mellifera, alors que les abeilles du Trentin se situent dans une position intermédiaire entre Apis mellifera ligustica et Apis mellifera carnica.
\end{abstract}

\section{INTRODUCTION}

Parmi les nombreuses races appartenant à l'espèce $A$ pis mellifera et présentes sur le territoire italien, Apis mellifera ligustica Spinola également appelée « abeille italienne $\gg$ a toujours été la plus répandue. D'autres races et leurs respectifs hybrides sont repérables, dont les localisations sont, à l'origine, limitées : Apis mellifera mellifera dans les zones de frontière avec la France et la Suisse, Apis mellifera carnica dans les zones de frontière avec l'Autriche et la Yougoslavie, Apis mellifera sicula restée dans quelques zones limitées de la Sicile; dans les autres parties de l'île, à cause des importations importantes d'essaims d'abeilles et de reines du continent, l'état d'hybridation est très répandu.

Aujourd'hui Apis mellifera ligustica, à l'état pur ou hybridé avec d'autres races, est présente dans presque tous les continents, pour la raison également que les qualités biologiques de ses reines sont à l'origine d'une demande importante des pays du monde entier depuis la moitié du siècle dernier. 
Les études visant à connaître et caractériser morphologiquement et biologiquement la race qui peuple le territoire italien sont d'un grand intérêt dans la mesure où elles constituent la base de tout travail de sélection.

En Italie Grassi (1881) a mené les premières études sur ce thème, qui se basait principalement sur la détermination de la couleur et des dimensions. Les études biométriques furent commencées par Vecchi et Giavarini (1938, 1950) et Giavarini (1956) qui ont pris en considération les caractères de la tête, des pattes, des ailes et de l'abdomen. Giavarini (1954) a étendu sa recherche également à l'A pis mellifera sicula, en examinant non seulement les ouvrières mais aussi les faux-bourdons. Prota (1976) a commencé une étude biométrique sur les populations d'Apis mellifera qui sont présentes en Sardaigne. Pour les zones de la chaîne des Alpes, des études ont été entreprises par Bolchi SerinI et al. (1983) qui ont pu constater, grâce aux méthodes de statistique univariée, une variabilité considérable entre et à l'intérieur des échantillons. L'analyse multivariable pour l'élaboration des données a été utilisée récemment par nous afin de vérifier l'existence d'un gradient d'altitude entre les échantillons pris dans les différentes zones de l'Italie centrale-septentrionale.

Cette étude représente la première partie d'un sondage systématique qu'on a prévu de faire sur la population d'abeilles du territoire italien, dans le but d'approfondir les connaissances sur l'abeille ligustica, localiser les zones où elle peut encore être trouvée à l'état pur, étudier la variabilité entre les races et déterminer les caractères morphologiques les plus appropriés à ces buts. Pour caractériser $A$. mellifera ligustica, les hybrides et écotypes éventuels par comparaison avec les races voisines, l'étude a été étendue également à des échantillons provenant de la France et de l'Autriche. On a souhaité ainsi former une grille de base servant de repère pour la classification des colonies inconnues (CORNUET et al., 1975).

\section{MATERIEL ET METHODES}

\section{A. Prise des échantillons}

Le sondage a été entreprise en considérant trois zones de l'Italie septentrionale (fig. 1) : la première à la frontière avcc la France (Ligurie occidentale - Province de Impéria), la seconde située dans la zone de frontière avec l'Autriche (Trentin - Haut-Adige - Province de Trento) et la troisième située loin des frontiè:es, avec moins de possibilités donc d'être concernée par des phénomènes dhybridation (Emilie Romagne - Provinces de Bologne, Modène, Forli).

Au cours du printemps 1982, 10 échantillons d'ouvrières ont été pris pour chaque zone et pour autant de ruches appartenant à des ruchers entre eux éloignés. Suivant les données fournies par les propriétaires, ces ruches étaient «originaires» du lieu, permanentes, éloignées de zones faisant l'objet de nomadisme, les reines avaient toujours été remplacées de manière naturelle. 


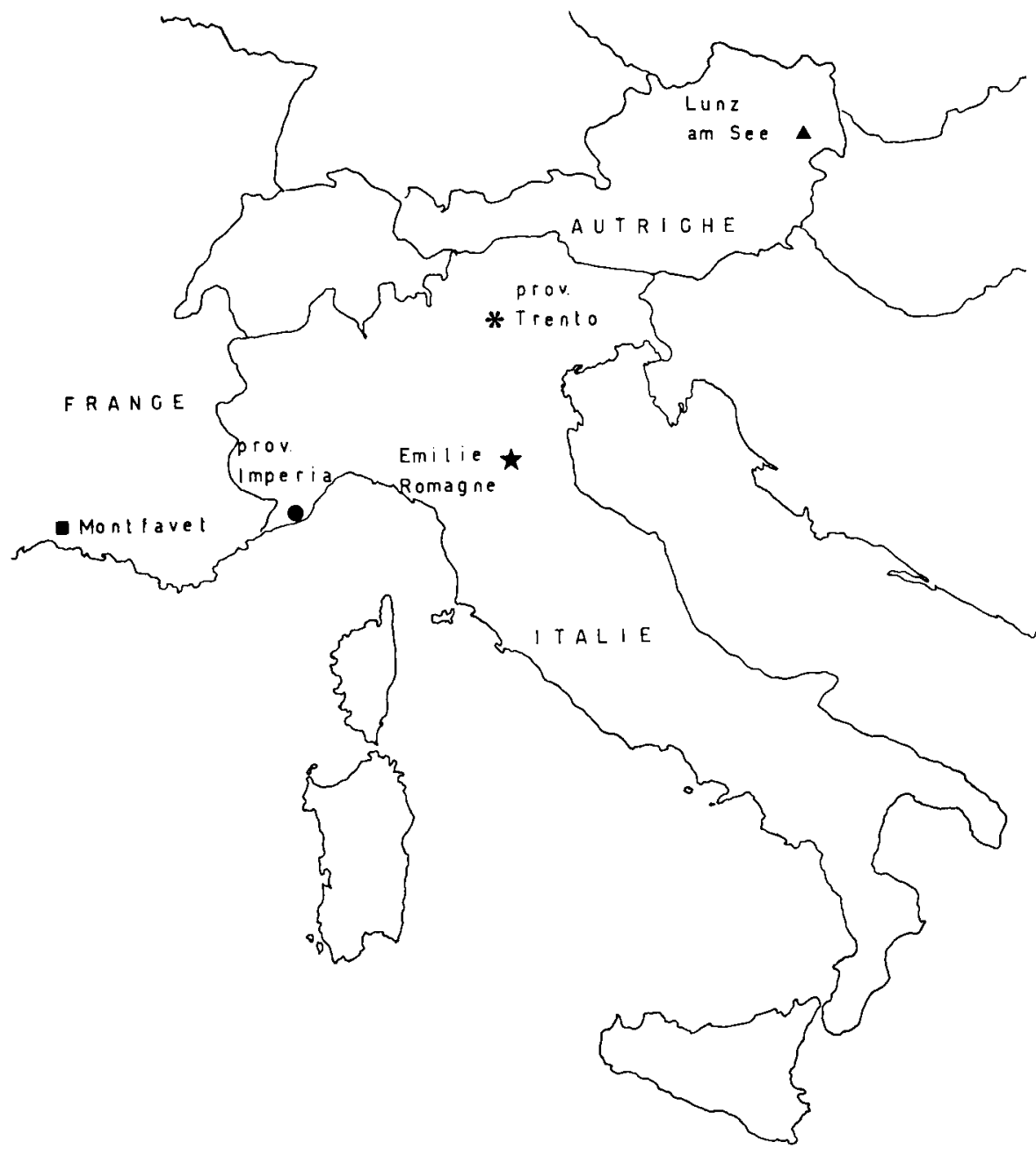

Fig. 1. - Répartition des cinq zones de prélèvement dans le secteur géographique choisi.

Fig. 1. - Distribution of the five zones of sampling in the geographic areas chosen.

Les 20 autres échantillons provenaient de 2 zones dont une était située dans la région de distribution d'Apis mellifera mellifera (France - Montfavet) et l'autre d'Apis mellifera carnica (Autriche - Lunz am See).

Au total on a pris 50 échantillons.

Toutes les abeilles ont été tuées par évaporation d'acétate d'éthyle $\left(\mathrm{CH}_{3} \mathrm{COOC}_{2} \mathrm{H}_{5}\right)$ et placées ensuite en liquide de conservation (Fampell's) jusqu'au moment de leur utilisation. 
TABL. 1. - Liste des caractères morphologiques étudiés

TABL. 1. - List of the morphological characters studied

\begin{tabular}{|c|c|c|}
\hline $\mathbf{N}^{0}$ & $\begin{array}{l}\text { Caractères } \\
\text { Characters }\end{array}$ & $\begin{array}{l}\text { Unité de mesure } \\
\text { Unit of measure }\end{array}$ \\
\hline 1 & $\begin{array}{l}\text { Largeur du tomentum sur le } 4^{*} \text { tergite } \\
\text { Width of tomentum on the } 4 \text { th tergite }\end{array}$ & $\mathrm{mm}$ \\
\hline 2 & $\begin{array}{l}\text { Longeur des poils sur le } 5^{c} \text { tergite } \\
\text { Length of hairs on the } 5 \text { th tergite }\end{array}$ & $\mathrm{mm}$ \\
\hline 3 & $\begin{array}{l}\text { Largeur de la bande jaune sur le } 2^{\circ} \text { tergite } \\
\text { Width of the yellow strip on the } 2 \text { nd tergite }\end{array}$ & $\mathrm{mm}$ \\
\hline 4 à 6 & $\begin{array}{l}\text { Segments } A \text { et } B \text { de la } 3^{\circ} \text { cellule cubitale et leur rapport } \mathbf{A} / \mathbf{B} \text { (index } \\
\text { cubital) (Fig. 1) }\end{array}$ & $\mathrm{mm}$ \\
\hline & $\begin{array}{l}\text { Segments } A \text { and } B \text { of the } 3 \text { rd cubital cell and their ratio } A / B \text { (cubital } \\
\text { index) (Fig. 1) }\end{array}$ & \\
\hline 7 à 12 & $\begin{array}{l}\text { Côtés des deux triangles formés par les lignes reliant les points de } \\
\text { rencontre des nervures de l'aile antérieure droite (Fig. 1) }\end{array}$ & $\mathrm{mm}$ \\
\hline & $\begin{array}{l}\text { Sides of both triangles made by the lines joining the meeting points of the } \\
\text { right front wing veins (Fig. i) }\end{array}$ & \\
\hline \multirow[t]{2}{*}{13 à 15} & $\begin{array}{l}\text { Trois angles (A } 4, \mathrm{~B} 4, \mathrm{~L} 13 \text { ) compris dans les triangles mentionnés } \\
\text { (Fig. 1) }\end{array}$ & degrés \\
\hline & Three angles (A 4, B 4, L 13) between the three triangles mentioned (Fig. 1) & degrees \\
\hline 16 à 17 & $\begin{array}{l}\text { Longueur et largeur de l'aile antérieure droite (Fig. 1) } \\
\text { Length and width of the right front wing (Fig. 1) }\end{array}$ & $\mathrm{mm}$ \\
\hline 18 & $\begin{array}{l}\text { Longueur du } 3^{e} \text { sternite } \\
\text { Length of the } 3 \text { rd sternite }\end{array}$ & $\mathrm{mm}$ \\
\hline 19 & $\begin{array}{l}\text { Scutellum } \\
\text { Scutellum }\end{array}$ & elasses $0-9$ \\
\hline 20 & $\begin{array}{l}\text { Scutum-postnotum } \\
\text { Scutum-postnotum }\end{array}$ & classes $0-7$ \\
\hline
\end{tabular}

\section{B. Caractères biométriques et méthodes de mesure}

Pour cette recherche (tableau 1) 6 caractères biométriques $\left({ }^{*}\right)$ ont été choisis, qui sont, selon nombreux auteurs (Goetze, 1963; Alpatov, 1935; Fresnaye, 1965; RuttNer, 1968; Tomassone et Fresnaye, 1971; Cornuet et al., 1975, et Cornuet et al., 1978) parmi les plus significatifs pour la différenciation des races européennes (1-6). A ces caractères, d'autres ont été ajoutés, qui avaient été utilisés précédemment (13-20) (AlPatov, 1929, 1935; GoEtze, 1930 et RutTNER, 1978), ou qui avaient été retenus pour la première fois (7-12). Les caractères mesurés sur l'aile sont repris dans la figure 2 .

$(\%$ Le caractère de la longueur de la proboscis n'a pas été retenu, car il fera l'objet de recherches détaillées ultérieures. 

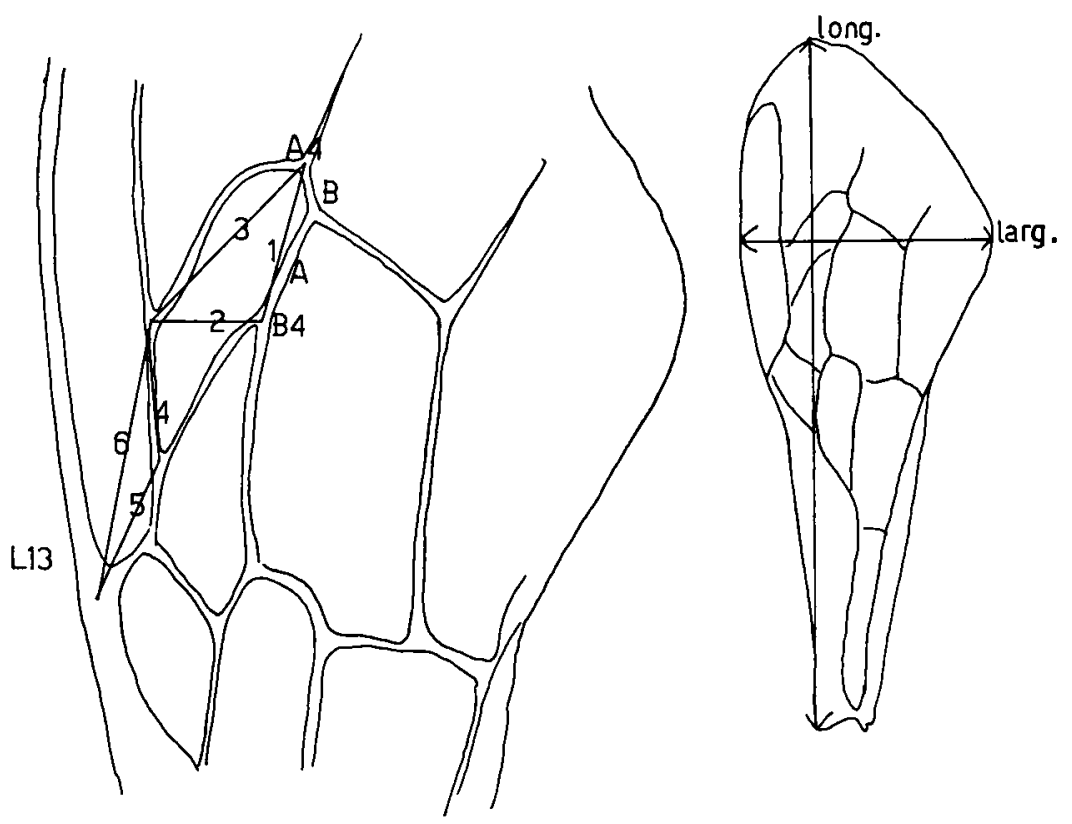

FIG. 2. - Caractères mesurés sur l'aile.

FIG. 2. - Wing characters measured.

Les abeilles ont été préparées en appliquant et utilisant la méthodologie décrite par FresNaYE (1981).

Les caractères 1-3, 16-20 ont été évalués au moyen d'un microscope à oculaire micrométrique ; pour les caractères restants 4-14 un microprojecteur a été utilisé. Les unités de mesure utilisées pour les divers caractères sont indiquées au tableau 1. Les mesures ont été effectuées sur 20 abeilles ouvrières pour chaque échantillon, suivant la méthode «abeille par abeille (FrESNAYE, 1981).

\section{Méthodes statistiques}

Pour chaque colonie étudiée on a calculé la moyenne, l'écart-type et les limites maximum et minimum des valeurs de chaque variable.

Pour chaque variable on a effectué les distributions relatives de fréquences à l'intérieur de chaque zone.

Le pouvoir discriminant des différents caractères a été évalué suivant l'analyse de la variance. Les composantes de variabilité entre zones ont été reportées sous forme de pourcentage de chacune des composantes par rapport au total. Par le test $F$ on a vérifié la signification de la variance entre les zones par rapport à celle calculée entre les ruches à l'intérieur de chaque zone.

La comparaison entre les populations, faite en tenant compte de tous les différents caractères ou groupes de caractères. a été effectuée selon les techniques d'analyse multivariable (analyse factorielle discriminante), qui a été décrite par Tomassone et Fresnaye (1971). 


\section{RÉSULTATS}

\section{A. Caractéristiques biométriques des populations}

Les distributions de fréquence à l'intérieur de la même zone de tous les caractères examinés résultent être unimodales, à l'exception de celle qui se rapporte au caractère couleur, dont l'allure est bimodale dans la population du Trentin; dans la plupart des échantillons (6 ruches sur 10 ) de cette zone des abeilles ont une couleur jaune très limitée ou en tâches du $2^{\mathrm{e}}$ tergite (de 1 à 10 abeilles sur un total de 20 mesurées pour chaque ruche). Par contre, l'histogramme de la population de Ligurie ne peut être considéré bimodal, car les abeilles jaunes sont trop peu nombreuses. D'autre part le caractère scutum-postnotum révèle une discontinuité de distribution dans tous les groupes, provenant probablement du type de mesure (évaluation combinée de 2 caractères). A la figure 3 figurent les distributions de fréquence de la couleur dans les 5 zones, de par leur particularité.

Au tableau 2 figurent les valeurs moyennes, minimums, maximums et les écarts-type des mesures effectuées pour chacune des 5 zones. Les différences entre les groupes suivent une allure différente en fonction des caractères retenus. Certains caractères en effet (couleur, scutellum) sont propres et caractéristiques des populations de la province de Trento et de l'Emilie-Romagne et les différencient des autres, qui ont des valeurs moins élevées; d'autres caractères semblent être efficaces pour distinguer selon un gradient Est-Ouest les échantillons pris dans la zone la plus orientale (province de Trento, Emilie-Romagne, Autriche) des autres (France, province de Impéria) dont les valeurs sont tantôt supérieures (B, angle A 4, côtés 4 et 6 , pilosité), tantôt inférieures (A, index cubital, angle B 4, côtés 1, 2, 3) par rapport aux premiers ; d'autres caractères encore (longueur et largeur de l'aile, longueur du $3^{*}$ sternite) sembleraient plutôt différencier entre elles les zones orientales (Autriche, province de Trento, Emilie-Romagne) avec une réduction graduelle des dimensions passant du Nord au Sud.

Au tableau 2 figurent aussi les pourcentages des composantes de variance relatives aux différences entre les zones en ce qui concerne les différents caractères. Tous les caractères retenus ont une variance entre les zones significativement plus élevée que la variance entre les ruches, c'est pourquoi les valeurs du Test F n'ont pas été reportées dans le tableau. A l'exception du tomentum, les caractères normalement considérés comme importants dans le diagnostic de différenciation des races européennes (couleur, A, B, index cubital, pilosité) ont une composante de variance entre les zones très élevée, ce qui reconfirme leur validité. Une signification non négligeable a également d'autres mesures de l'aile (côtés 3 , 4,6 ) et le scutellum. 

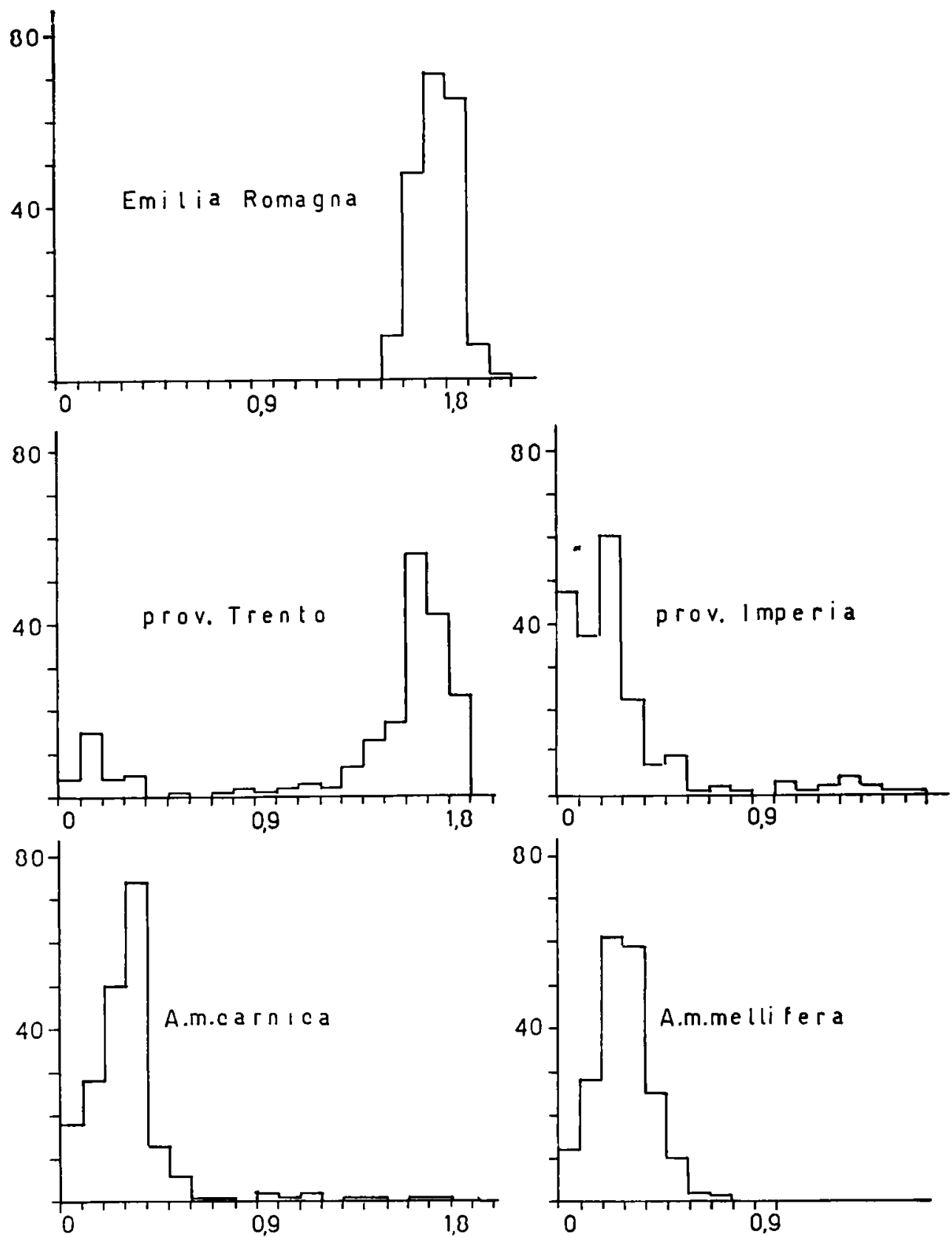

Fig. 3. - Histogrammes de la couleur des cinq populations étudiées.

En abscisses : largeur (en $\mathrm{mm}$ ) de la bande jaune sur le $2^{\text {e }}$ tergite.

En ordonnées : nombre d'abeilles.

FIG. 3. - Color histogram of the five populations studied.

$\mathrm{X}$-axis : width (in $\mathrm{mm}$ ) of the yellow strip on the 5 th tergite.

$Y$-axis : number of honeybees. 


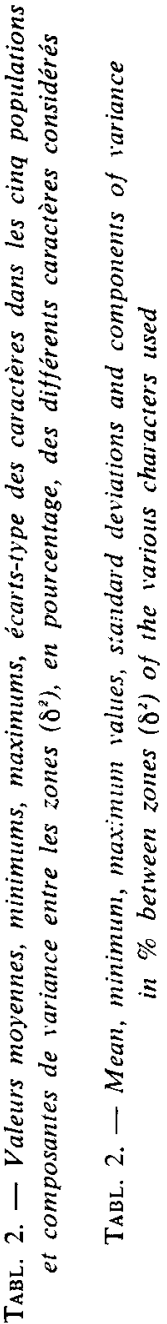

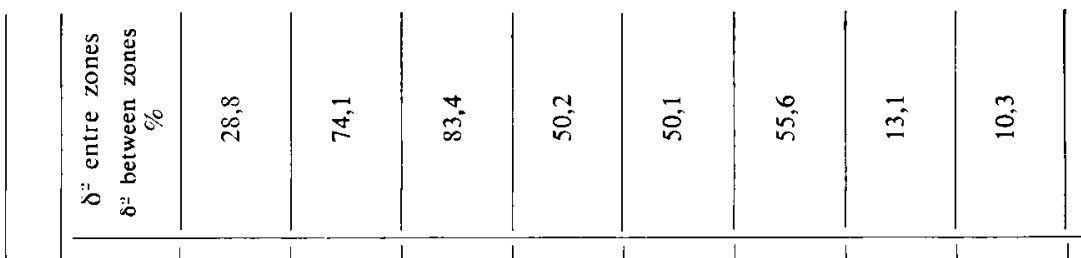

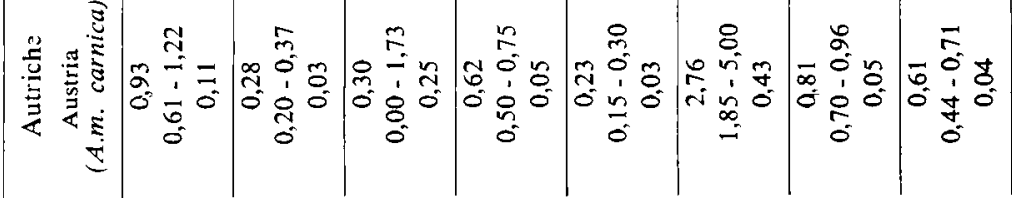

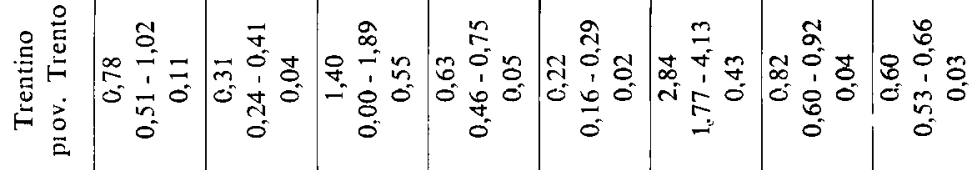

$$
\begin{aligned}
& \text { 㫄 }
\end{aligned}
$$

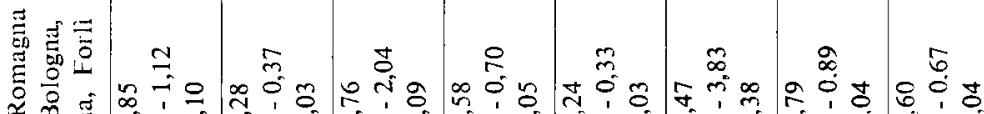

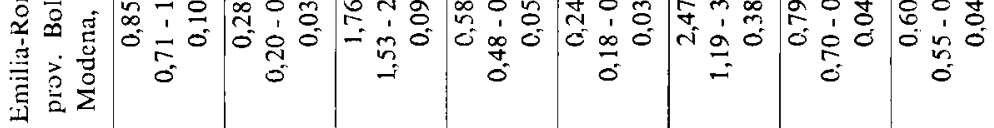

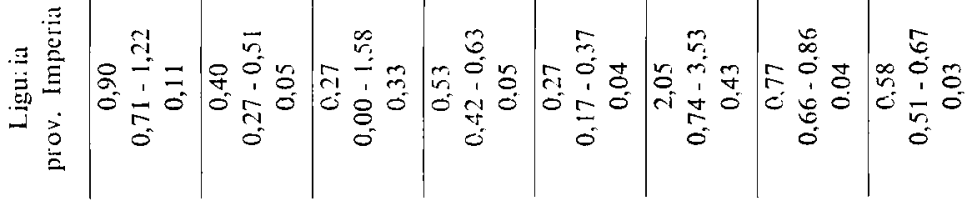

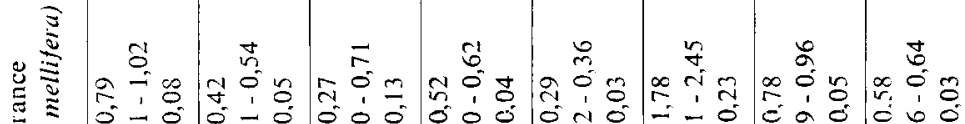

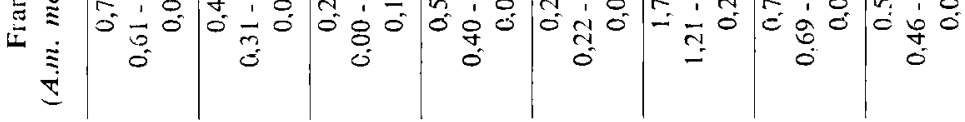

$$
\begin{aligned}
& \mid \\
& \stackrel{\stackrel{ \pm}{0}}{\stackrel{0}{ \pm}}
\end{aligned}
$$

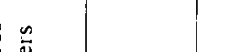

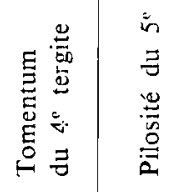

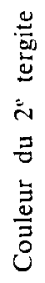

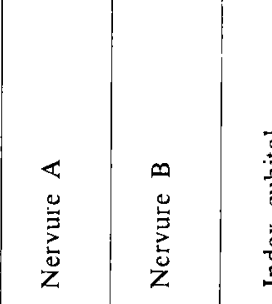

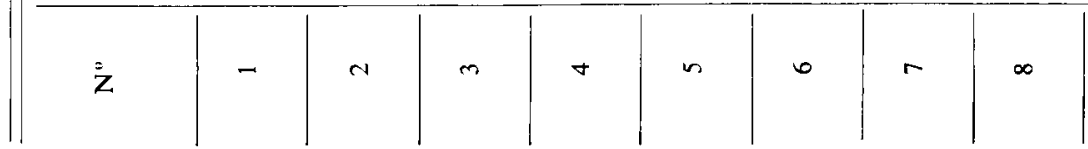




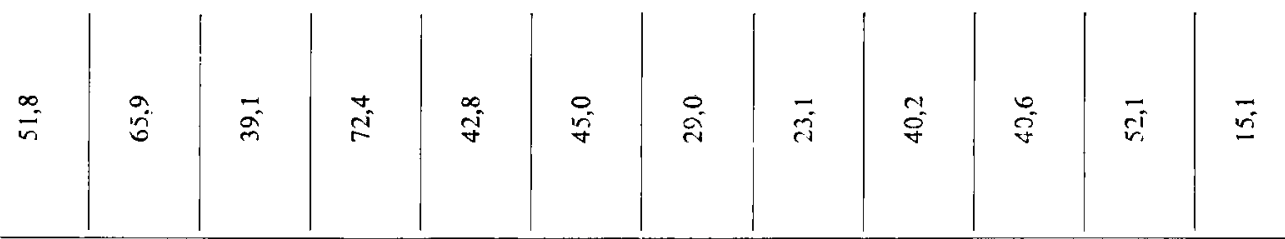

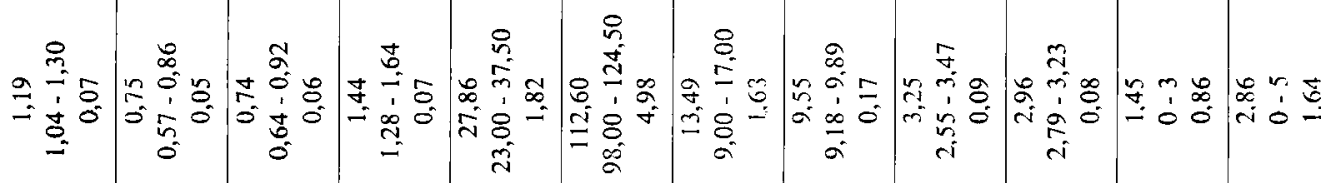

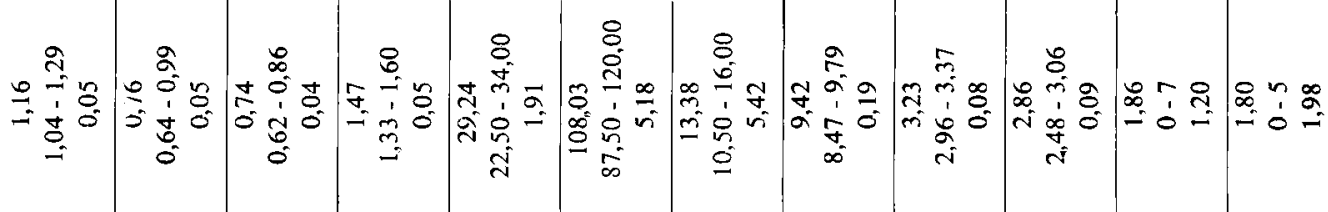

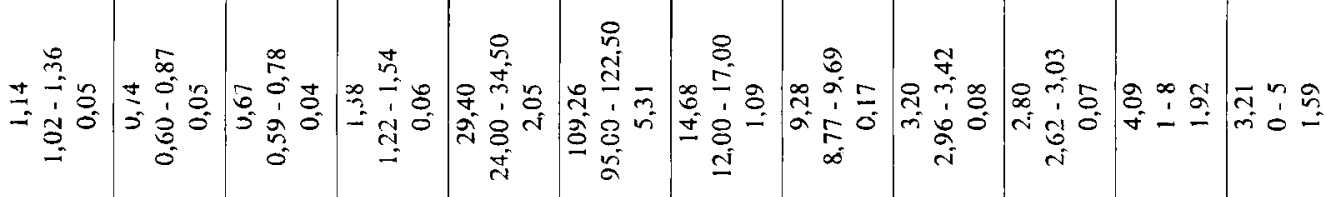

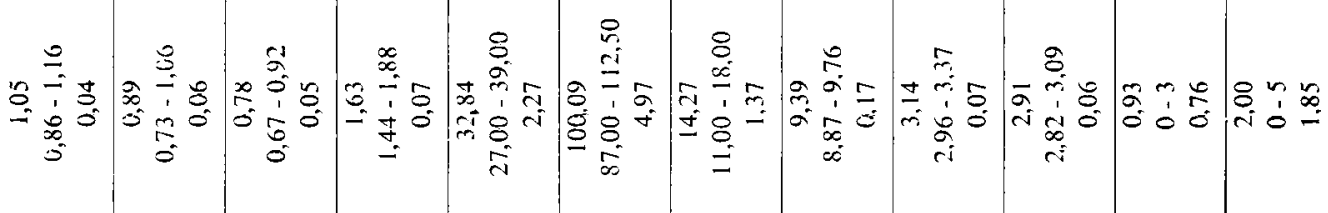

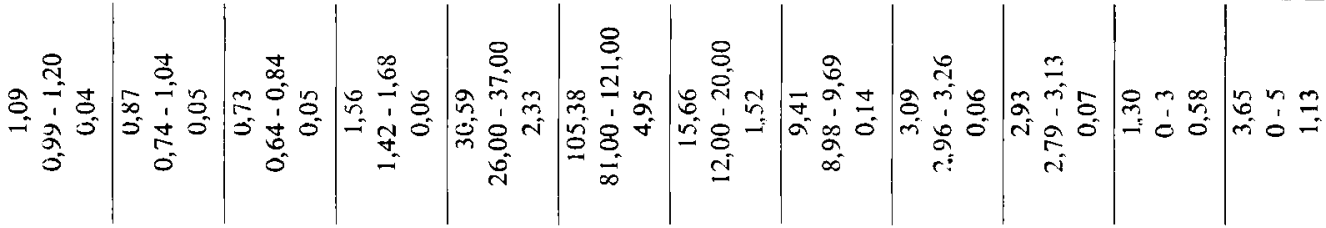

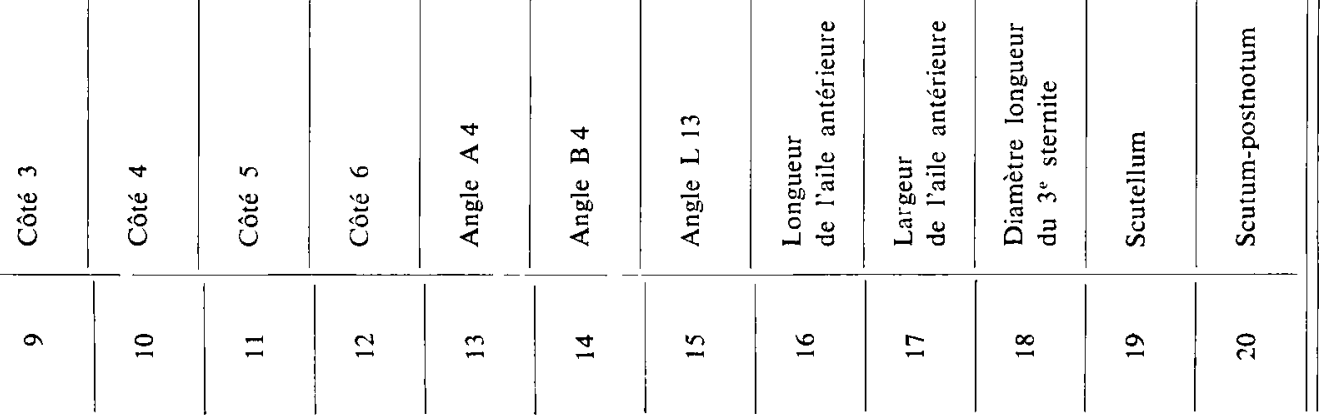


B. Discrimination des populations (analyse factorielle discriminante)

Pour toutes les analyses, il ressort que les 5 populations sont réparties suivant deux directions : ligne Est-Ouest, séparant France-Impéria de Autriche TrentoEmilie-Romagne ; ligne Nord-Sud, sur laquelle sont alignées Autriche, Trento et Emilie-Romagne ; ces gradients correspondent aux axes 1 et 2 des analyses factorielles discriminantes respectivement.

Dans la première analyse factorielle (Fig. 4), effectuée sur tous les caractères (à l'exception de l'index cubital), la discrimination entre les 5 zones s'exprime principalement sur les axes 1 et 2 . Le $1^{\text {"r }}$ axe donne une séf aration nette, suivant un gradient Est-Ouest, des populations de France-Impéria, fort semblables entre elles, du groupe des autres.

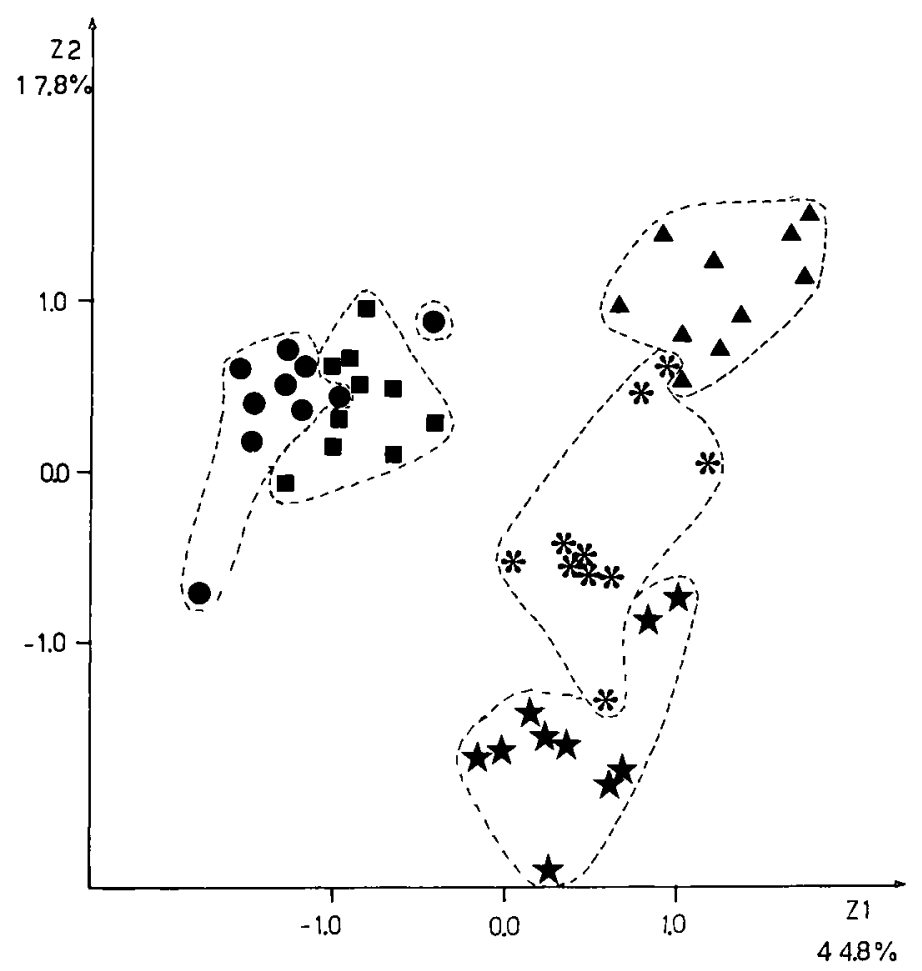

Fig. 4. - Représentation graphique de l'analyse sur tous les caractères (sans index cubital).

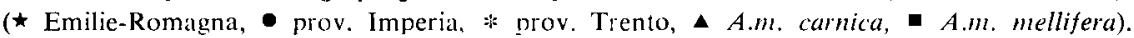

FIG. 4. - Graphics of the analysis upon all characters (except cubital index).

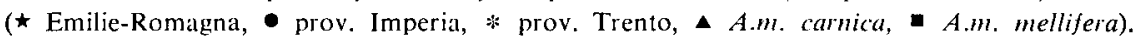


Le $2^{\circ}$ axe aligne, suivant un gradient latitudinal, les populations d'Autriche, de la province de Trente, de l'Emilie-Romagne.

L'analyse factorielle (Fig. 5) effectuée sur les caractères classiques considérés comme indicateurs de différences raciales (tomentum, A, B, pilosité, couleur) laisse inchangées les distances entre tous les groupes, à l'exception de Trento et Emilie-Romagne, qui révèlent une superposition assez marquée. Cette dernière situation doit propablement être attribuée au caractère couleur qui, de par sa distribution bimodale, tend à camoufler d'éventuelles différences entre les groupes par une coloration semblable (jaune ou noire). Cet effet a un poids encore plus important, compte tenu du nombre très limité de caractères utilisés pour cette analyse.

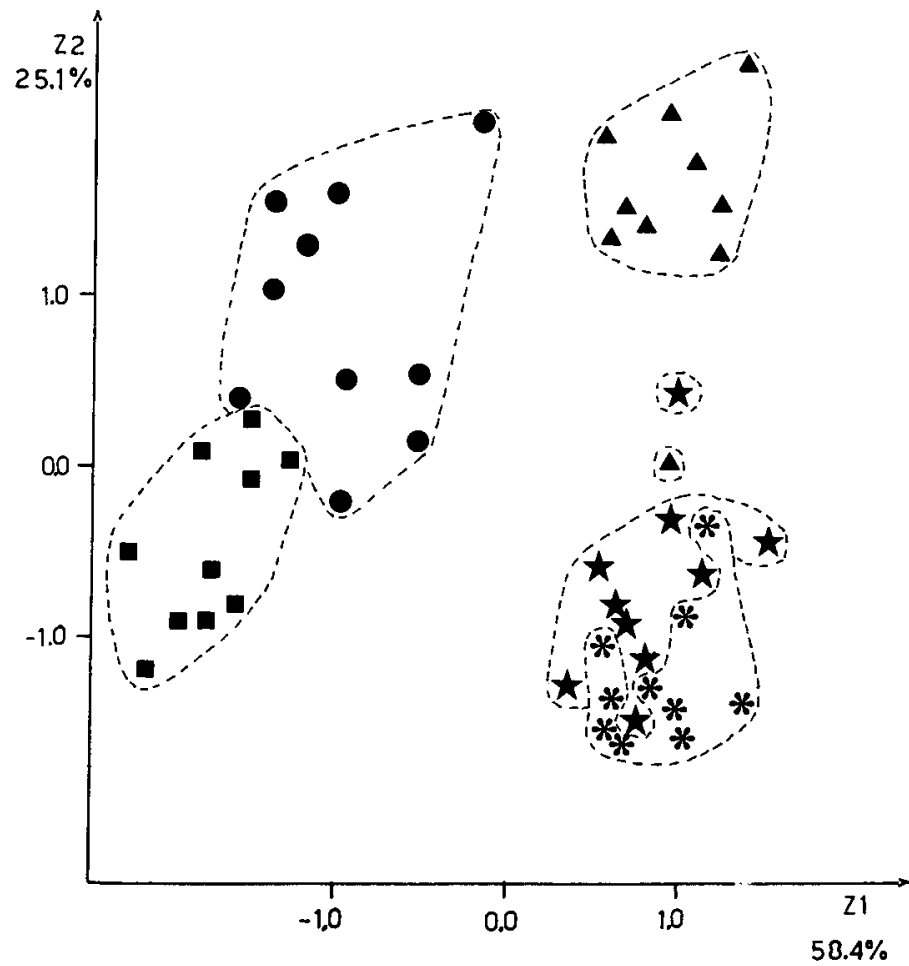

FIG. 5. - Représentation graphique de l'analyse sur cinq caractères raciaux.

( Emilie-Romagna, $\bullet$ prov. Imperia, * prov. Trento, ^ A.m. carnica, A.m. mellifera).

Fig. 5. - Graphics of the analysis upon 5 racial characters.

(Same symbols as in fig. 4). 
Si en fait d'autres caractères sont inclus dans l'analyse (côtés $3,4,6$ et scutellum) qui jouent, comme on a vu, un rôle important dans la différenciation des zones (Fig. 6), la population du Trentin se situe de nouveau dans une posisition intermédiaire entre les populations autrichienne et émilienne.

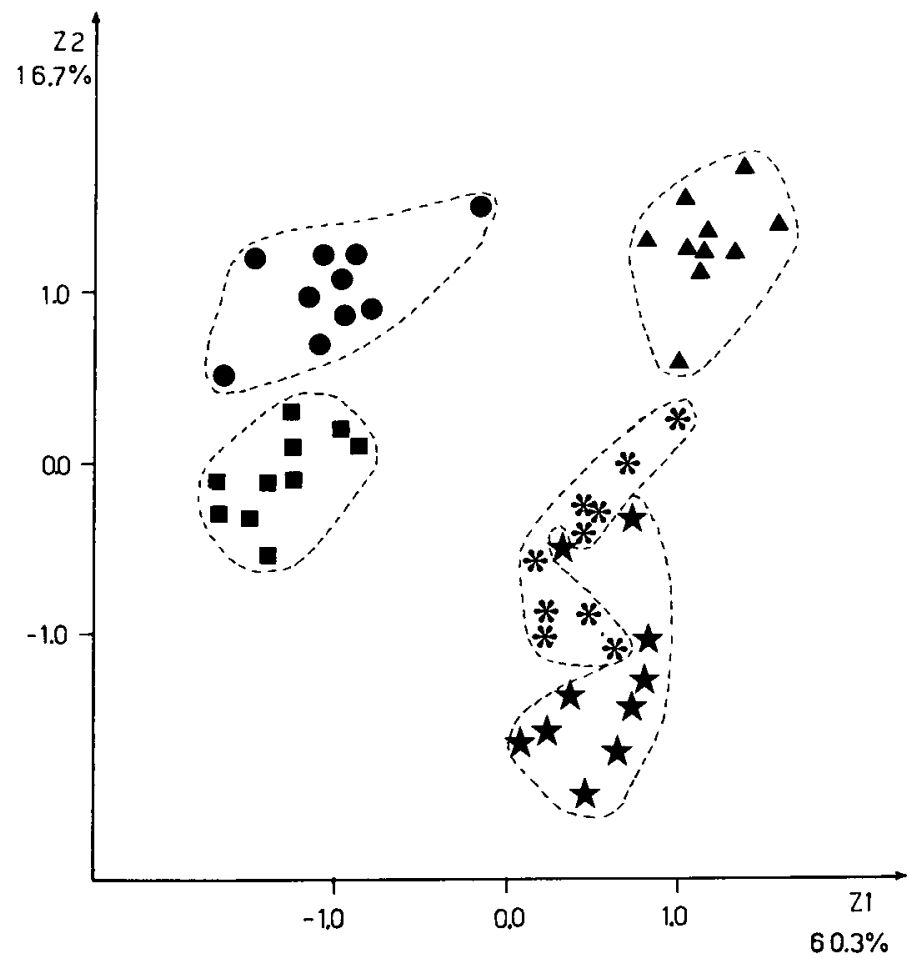

F1G. 6. - Représentation graphique de l'analyse sur neuf caractères raciaux. ( Emilie-Romagna, $\bullet$ prov. Imperia, * prov. Trento, ^A.m. carnica, - A.m. mellifera).

FIG. 6. - Graphics of the analysis upon 9 characters. (Same symbols as in fig. 4).

Dans l'échantillon du Trentin cependant les caractères retenus dans l'analyse précédente ne présentaient pas tous des valeurs intermédiaires par rapport à ceux des deux races voisines; une analyse (Fig. 7) effectuée uniquement sur ces caractères (tomentum, $\mathrm{A}, \mathrm{B}$, côtés 4 et 6 , pilosité) révélait en fait une position anormale, ce qui indique probablement une dérive génétique. 


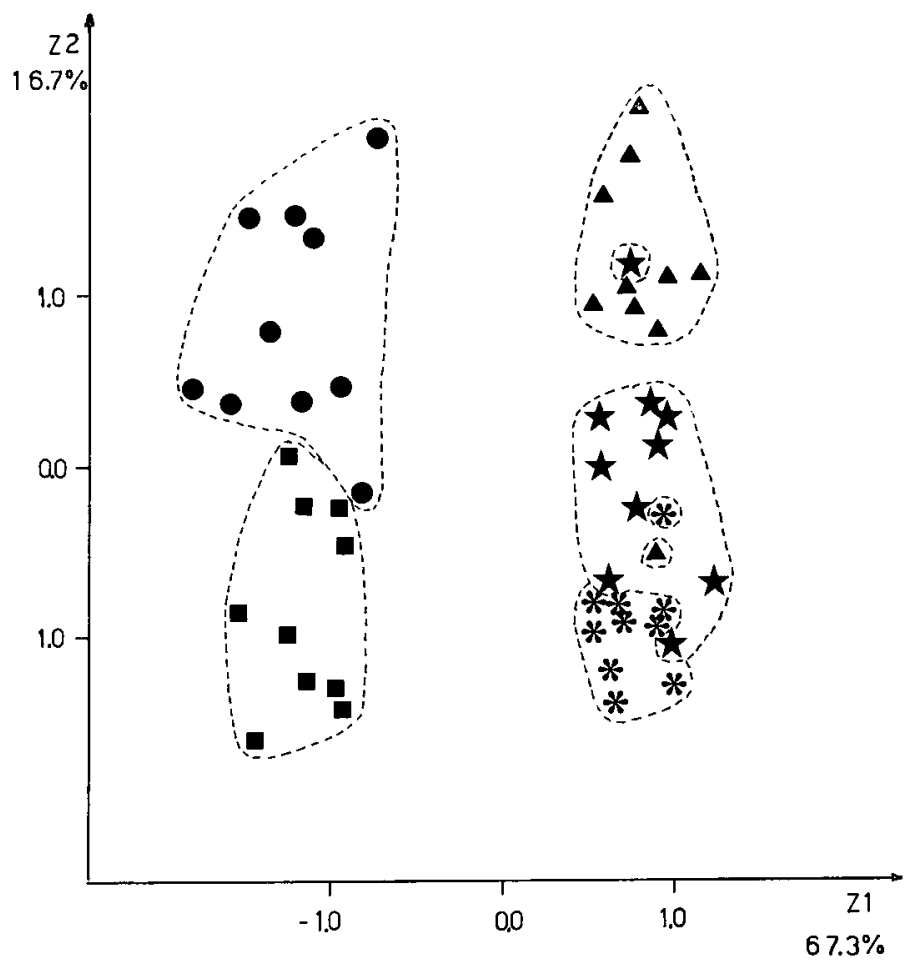

FIG. 7. - Représentation graphique de l'analyse pour l'étude de la dérive génétique du Trentin.

(* Emilie-Romagna, • prov. Imperia, * prov. Trento, A A.m. carnica, - A.m. mellifera).

FIG. 7. - Graphics of the analysis for the study of genetic drift in Trentin.

(Same symbols as in fig. 4).

La dernière analyse (Fig. 8), effectuée sur le groupe des caractères considérés comme de moindre importance (côtés 1, 2, 5 ; angles A 4, B 4, L 13 ; longueur et largeur de l'aile ; longueur du $3^{\circ}$ sternite ; scutum-postnotum) dans la différenciation des races européennes, remet en cause, même avec une plus forte variabilité, la discrimination déjà observée entre les groupes, suivant les gradients du milieu Est-Ouest et Nord-Sud. 


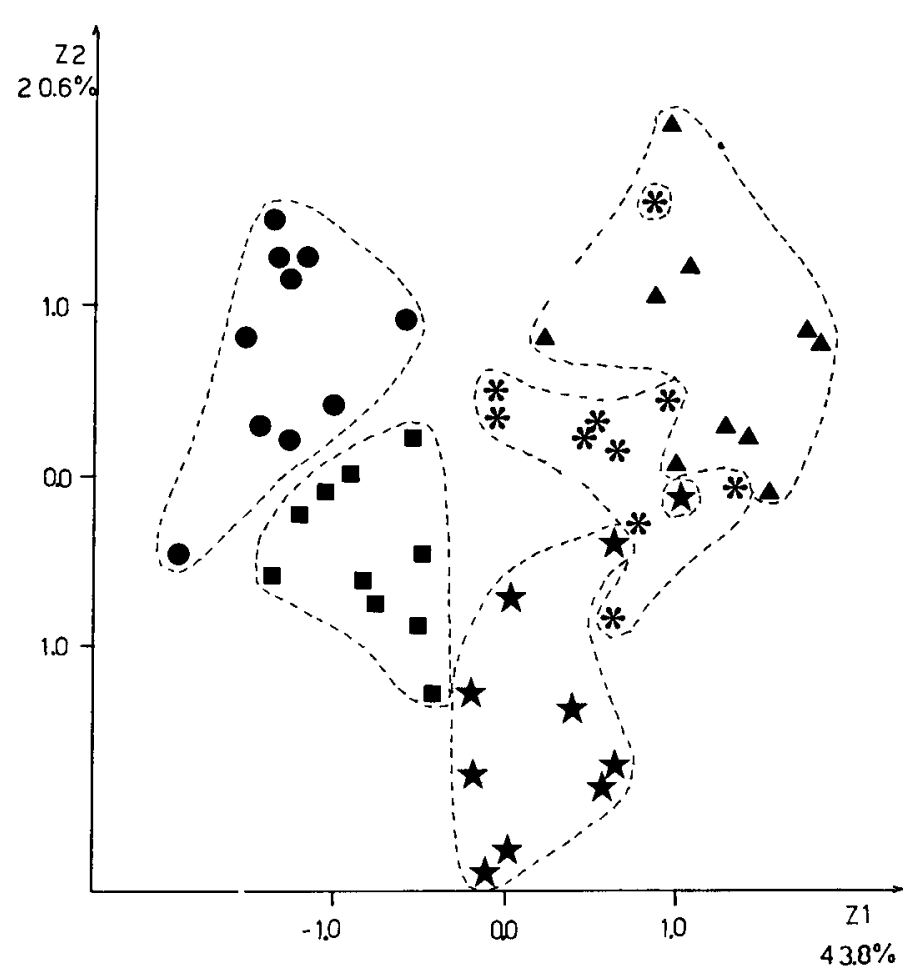

FIG. 8. - Représentation graphique de l'analyse sur les caractères raciatex les moins importants.

( Emilie-Romagna, - prov. Imperia, * prov. Trento, \ A.m. carnica, - A.m. mellifera).

FIG. 8. - Graphics of the analysis upon the less important racial characters. (Same symbols as in fig. 4).

\section{DISCUSSIONS ET CONCLUSIONS}

Tout en soulignant que les résultats obtenus et repris précédemment sont conditionnés par les modes de prise d'échantillons et par les zones choisies, l'étude menée sur les abeilles de l'Italie septentrionale ont donné une réponse positive et suffisamment représentative.

Les premières analyses statistiques (Tab. 2) montrent que tous les caractères examinés comportent une efficacité différente dans la différenciation des zones. Aucun n'a une valeur déterminante dans la définition des populations. Les populations ne peuvent apparaître suffisamment caractérisées et distinctes qu'en menant les analyses factorielles discriminantes sur les différentes combinaisons de caractères qui reproposent et complètent les résultats des analyses préliminaires. Pour toutes les analyses un résultat commun est la séparation nette des échantillons 
de A.m.mellifera, A.m. carnica des échantillons pris en Emilie-Romagne, ce qui confirme la présence de $A$.m. ligustica dans cette zone. Les échantillons de la Ligurie occidentale se situent en général assez près des échantillons français et cette similitude n'est pas étonnante, compte tenu du fait que ces deux zones sont proches et que les barrières naturelles sont faibles (cfr. BADINO, 1982).

Une interprétation différente peut être fournie pour la position intermédiaire de la population de la province de Trente qui se situe entre la population autrichienne et celle de l'Emilie pour certaines analyses (Fig. 4, 6, 8). Comme les abeilles carnica et ligustica «pures» peuvent être distinguées sur la base de leur couleur (RIHAR, 1961), la présence d'abeilles avec une large bande jaune

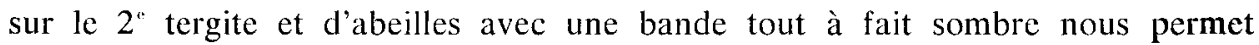
d'exprimer l'hypothèse de l'hybridation (*) (cfr. Bolchi SERINI, 1982), qui pourrait avoir concerné la zone tout entière, du fait que les abeilles noires sont présentes en 6 ruches sur 10 . On sait que le caractère couleur a sa base génétique avec une expression de type additif (Roberts, 1951). Comme les abeilles les plus sombres ne sont pas nombreuses dans les différentes ruches, on peut présumer une faible disjonction des gènes carniques dans la population trentine examinée.

D'ailleurs, certains caractères de cette population (Fig. 7) ne sont pas intermédiaires par rapport à ceux des populations autrichienne et italienne. Comme les hybrides révèlent habituellement des caractéristiques dans la gamme des races parentales (FRESNAYE, 1981) on peut envisager une dérive génétique pour certains caractères. L'hybridation devrait donc avoir eu lieu à des temps suffisamment lointains, pour permettre cette évolution. En partant de ces bases, nous pensons que l'on peut parler d' «écotype trentin» dont l'origine se situe dans une hybridation ancienne. L'existence de gradients du milieu de répartition des 5 populations se rattache à des études précédentes d'autres auteurs qui préconisaient une chaîne de races sahariensis-intermissa-iberica-mellifera et un autre groupe carnica-ligustica (RUTTNER, 1973 ; RutTNER et al., 1978 ; CORNUET et al., 1975 ; CORNUET et al., 1982).

Pour conclure, on a pu donc déterminer, au nombre des 20 caractères examinés, ceux qui sont les plus efficaces dans la différenciation raciale, et qui sont le moins affectés par le milieu, et pour lesquels la détermination génétique dépend d'un nombre restreint de facteurs. Toutes les combinaisons de caractères examinées suivant l'analyse factoriclle discriminante ont donné des résultats concordants pour la différenciation, même si c'est à un degré différent, des cinq zones de prélèvement, suivant les gradients latitudinaux et longitudinaux.

(*) On peut exclure l'hypothèse d'hybridation avec A.m. mellifera, noire elle aussi, car l'échantillon du Trentin se situe, pour toutes les analyses factorielles, le long du gradient longitudinal Autriche-Emilie Romagne, et qu'il est tout à fait séparé le long de l'axe 2 par rapport au groupe France-Ligurie. 
Des trois populations prises sur le territoire italien, la population émilienne seule peut être considérée ligustica. Les abeilles prises en Ligurie occidentale sont fort semblables aux abeilles françaises et leur emplacement est difficile à déterminer, car les échantillons de référence sur la côte tyrrhénienne sont absents. La population du Trentin, à notre avis, tire probablement son origine d'un processus d'hybridation très ancien et, sous l'effet de la sélection naturelle, elle va prendre les caractères d'un écotype.

Reçu pour publication en mars 1984. Accepté pour publication en juin 1984

\section{REMERCIEMENTS}

Nous adressons nos plus vifs remerciements à M. le Professeur F. Ruttner (Lunz Am See, Autriche) et J. Fresnaye (Monfavet, France) ainsi qu'aux apiculteurs (S. Sasso, F. MaccarI, G. Grovannini) qui ont contribué à la réalisation de ce travail par l'envoi d'échantillons d'abeilles.

\section{SUMMARY}

\section{BIOMETRIC STUDY OF THE GEOGRAPHIC VARIATION IN POPULATIONS OF APIS MELLIFERA L. IN NORTHERN ITALY}

Samples of bees from three different areas of Northern Italy (center-north, western border area, north-eastern border area) were examined, as well as samples of Apis mellifera mellifera (of French origin) and of Apis mellifera carnica (of Austrian origin) which were used for purposes of comparison (fig. 1).

Ten samples from different apiaries were taken in each area; 20 characteristics were measured on 20 bees of each sample, using the «bee by bee» method (tab. 1, fig. 2).

The data were analysed using multivariate analysis techniques (discriminant factor analysis). From the 20 characteristics examined it was possible to identify some which were more effective as a means of demonstrating racial differences (Tab. 2). All the combinations of characteristics examined using discriminant factor analysis (Figs 4, 5, 6 and 8), allowed consistent distinction of the 5 sample areas according to their latitudinal and longitudinal position, albeit to different extents.

Of the three populations taken from Italy only that from Emilia showed that it belonged to the ligustica race. The bees taken from Western Liguria proved to be very similar to those of French origin and, in any case difficult to place because of the lack of reference samples from the Tyrrhenian ccast. The population from Trentino probably had its origins in a hybridation process of long ago and is assuming the characteristics of an ecotype (fig. 7) as a result of natural selection.

\section{ZUSAMMENFASSUNG}

\section{BIOMETRISCHE UNTERSUCHUNG DER GEOGRAPHISCHEN VARIABILITATT VON APIS MELLIFERA L. IN NORDITALIEN}

Es wurden Bienenproben aus drei verschiedenen Gebieten Norditaliens (zentrales Gebiet im Norden, westliche Grenzzone, nordöstliches Grenzgebiet) untersucht, außerdem Vergleichsproben von Apis mellifera mellifera aus Frankreich und von Apis mellifera carnica aus Österreich (Abb. 1). 
Aus jeder Zone wurden 10 Proben von verschiedenen Bienenvölkern entnommen. Von jeder Probe wurden nach der «Biene für Biene-Methode» an 20 Bienen je 20 Merkmale gemessen (Tab. 1, Abb. 2).

Die Daten wurden statistisch nach einer Multivariat-Analyse (Diskriminanzanalyse) verarbeitet. Unter den 20 untersuchten Merkmalen konnten diejenigen bestimmt werden, die für die Unterscheidung der Rassen die größte Bedeutung haben (Tab. 2).

Alle Merkmalskombinationen haben in der Diskriminanzanalyse (Abb. 4-8) für die Unterscheidung der fünf untersuchten Gebiete übereinstimmende Resultate ergeben, wenn auch in verschiedenem Ausmaß, je nach der Betrachtung in Richtung der geographischen Breite oder Länge.

Von den drei Populationen aus dem italienischen Territorium erwiesen sich nur die Proben aus der Emilia als zugehörig zur Rasse ligustica. Die Bienen aus Westligurien ähnelten sehr stark den französischen Bienen; wegen des Fehlens von Vergleichsproben von der tyrrhenischen Küste konnte jedoch ihr Verbreitungsgebiet nicht bestimmt werden. Die Populationen aus dem Trentino entstammen wahrscheinlich einer weit zurückliegenden Hybridisierung, die infolge natürlicher Selektion zu einem eigenen Ökotyp geführt hat (Abb. 7).

\section{RÉFÉRENCES BIBLIOGRAPHIQUES}

Alpatov W.W., 1929. - Biometrical studies on variation and races of the honeybee. Quart. Rev. Biol., 4, 1-58.

Alpatov W.W, 1935. - Sur les variations des abeilles. 3. - La cellule cubitale de l'aile du genre Apis du point de vue de la diagnose et de l'évolution. Zool. Zh., 14 (4), 664-672.

Badino G., Celebrano G. Manino A., 1982. - Genetic variability of Apis mellifera ligustica Spin. in a marginal area of its geographical distribution. Experientia, 38, 540-541.

Bolch i Serini G., Sommaruga A., Lapietra G., 1983, - Studio biometrico di popolazioni alpine di Apis mellifera L. In stampa.

Cornuet J.M., Fresnaye J., Tassencourt L., 1975. - Discrimination et classification de populations d'abeilles à partir de caractères biométriques. Apidologie, 6 (2), 145-187.

Cornuet J.M., Fresnaye J., Lavie P., 1978. - Etude biométrique de deux populations d'abeilles cévenoles. Apidologie, 9 (1), 41-55.

Cornuet J.M., Albisetti J., Mallet N., Fresnaye J., 1982, - Etude biométrique d'une population d'abeilles landaises. Apidologie, 13 (1), 3-13.

Fresnaye J., 1965. - Etude biométrique de quelques caractères morphologiques de l'abeille noire française (A.m. mellifica). Ann. Abeille, 8 (4), 271-283.

Fresnaye J., 1981. - Biométrie de l'abeille, 2" éd. Echauffour (Ornc), Office pour l'Information et la Documentation en Apiculture, $56 \mathrm{p}$.

Giavarini I., 1954. - Ricerche sui caratteri razziali dell'Apis mellifica ligustica Spinola (Studi biometrici e morfologici sui fuchi). Mem. Soc. entomol. ital,, 32, 119-128.

Giavarini I., 1954. - Ricerche biometriche e morfologiche sui caratteri razziali di Apis mellifica sicula Grassi. Mem. Soc. Entomol. Ital, 32, 129-139.

Giavarini I., 1956. - Studio comparativo sulle lamine paramerali e sulle valve del pene in fuchi di Apis mellifica ligustica Spinola ed in ibridi di questa con Apis mellifica mellifica $\mathbf{L}$. Apicolt. Italia, 23 (9-10), 199-200.

GraSsi B., 1881. - Saggio di una monografia delle api d'Italia. L'Apicoltore, 9, 277-280.

Goetze G., 1930. - Variabilitäts- und Züchtungsstudien an der Honigbiene mit besonderer Berücksichtigung der Langrüsseligkeit. Arch. Bienenkd., 11, 185-279. 
Goetze G., 1964. - Die Honigbiene in natülicher und künstlicher Zuchtatslese, 2 vol. Hamburg, P. Parey, $120+92 \mathrm{p}$.

Leporati M., Val.1. M., Cavicchi S., 1983. - Variazioni ambientali in popolazioni di Apis mellifera ligustica : analisi del potere discriminatorio di alcuni caratteri biometrici. Quad. Doc. F. A.I., 4, 20 pp.

Prota R., 1976. - Osservazioni sulla variabilità somatometrica delle poplazioni sarde di Apis mellifera ligustica Spinola. Apicoll. mod., 67, 77-81.

Ruhar J., 1961. - Recherches biométriques sur la couleur des abeilles carnioliennes en Yougoslavie. Ann. Abeille, 4 (1), 41-50.

Roberts W.C., Mackensen O., 1951. - Breeding improved honeybees. Am. Bee J., 91, 292-294, $328-330,382-384,418-421,473-475$.

Ruttner F., 1968. - Les races d’abeilles. In : Traité de biologie de l'abeillé, éd. R. Chauvin, t. 1. Paris, Masson, 27-44.

Rutriner F., 1973. - Die Bienenrassen des mediterranen Beckens. Apidologie, 4 (2), 181.

Ruttner F., Tassencourt L., Louveaux J., 1978. - Biometrical-statistical analysis of the geographic variability of Apis mellifera L., Apidologie, 9 (4), 363-381.

Tomassone R., Fresnaye J., 1971. - Etude d'une méthode biométrique et statistique permettant la discrimination et la classification de populations d'abeille (Apis mellifica L.). Apidologie, 2 (1), 49-65.

Vecchi A., Giavarini I., 1938. - Ricerche biometriche sull'Apis mellifica ligusticis. Atti VII Int. Kongr. Entomol, Berlin.

Vecchi A., Giavarini 1., 1950. - Ricerche sui caratteri razziali dell"ape domestica (Indice cubitale dell'ala e indice della piastra stigmatica dell' 8" urite). Boll. Ist. enomol. Univ. Bologna, 18, $1-13$. 